\title{
Clinical application of a new warfarin-dosing regimen based on the CYP2C9 and VKORC1 genotypes in atrial fibrillation patients
}

\author{
NIAN-XIN JIANG, JUN-WEI GE, YU-QIONG XIAN, SHAO-YING HUANG and YAN-SONG LI \\ Department of Cardiology, Shanghai Seventh People's Hospital, Shanghai 200137, P.R. China
}

Received August 31, 2015; Accepted December 7, 2015

DOI: $10.3892 / b r .2016 .617$

\begin{abstract}
The polymorphisms of cytochrome P450 2C9 (CYP2C9) and vitamin $\mathrm{K}$ epoxide reductase complex 1 (VKORC1) are important genetic factors for warfarin dose determinations. The present study aimed to investigate the contribution of the CYP2C9 and VKORC1 genotypes to warfarin dose requirement in atrial fibrillation (AF) patients, and to evaluate the clinical application of a warfarin-dosing algorithm. A total of 122 AF patients with a target international normalized ratio of 2.0 to 3.0 were included to determine the genotypes of CYP2C9 (rs1057910) and VKORC1 (rs9923231). A warfarin-dosing algorithm was developed based on age, height, and the CYP2C9 and VKORC1 genotypes of AF patients. The results indicated that the mean warfarin daily dose requirement was lower in the CYP2C9*1/3 3 genotype compared with those in the homozygous wild-type CYP2C9*1/*1 patients $(\mathrm{P}<0.05)$, and was higher in patients with the VKORC1 AG and GG genotypes compared with those with the AA genotype $(\mathrm{P}<0.05)$. The multivariate regression model showed that age, height, and the CYP2C9 and VKORC1 genotypes were the best variables for estimating warfarin dose $\left(\mathrm{R}^{2}=56.4 \%\right)$. A new warfarin-dosing algorithm was developed and its validity was confirmed in a second cohort of AF patients. During the 50-day follow-up, 63.3\% (19/30) of control group patients and $86.7 \%(26 / 30)$ of patients in the experimental group acquired the warfarin maintenance dose. Among all the patients who acquired the warfarin maintenance dose, the mean time elapse from initiation until warfarin maintenance dose was significantly less in the experimental group (25.8 \pm 1.7 day) compared to the control group $(33.1 \pm 1.9$ day $)(\mathrm{P}<0.05)$. There was significant linear correlation between predicted warfarin maintenance dose and actual dose $(\mathrm{r}=0.822, \mathrm{P}<0.01)$. In conclusion, a new warfarin-dosing algorithm was developed based on the CYP2C9 and VKORC1 genotypes, and it can
\end{abstract}

Correspondence to: Dr Yan-Song Li, Department of Cardiology, Shanghai Seventh People's Hospital, 358 Datong Road, Shanghai 200137, P.R. China

E-mail: liyssh@163.com

Key words: atrial fibrillation, warfarin, cytochrome P450 2C9, single nucleotide polymorphisms, vitamin $\mathrm{K}$ epoxide reductase complex 1 shorten the time elapse from initiation until warfarin maintenance dose in AF patients with warfarin therapy.

\section{Introduction}

Warfarin remains the most commonly prescribed oral anticoagulant drug $(1,2)$, and has long been applied in the prevention and treatment of various venous and arterial thromboembolic disorders, such as deep venous thromboembolism, pulmonary embolism, prosthetic heart valves and atrial fibrillation (AF) (3). Due to inter-individual variation of warfarin dosing, inadequate or excessive warfarin may result in thromboembolism or bleeding. Therefore, the warfarin dose should be monitored and controlled within a narrow therapeutic window in clinical application. In fact, in patients undergoing warfarin therapy, the prothrombin time (PT), expressed as the international normalized ratio (INR), was maintained within the therapeutic range, thereby providing effectiveness and safety of warfarin (4). A number of studies have shown that patients with an INR above the upper limit of the therapeutic range have an increased risk of bleeding $(5,6)$, and patients with an INR below the lower limit have an increased risk of thromboembolic events $(7,8)$.

The inter-individual variation of warfarin dose response is caused by various factors, such as patient demographics, environment, clinical background, and particularly, genetic polymorphism $(9,10)$. Therefore, the Food and Drug Administration proposed the genetic testing for estimating initial warfarin dose in 2007 (11). From then on, numerous studies have reported a variety of genetic polymorphisms in genes associated with the warfarin pharmacodynamics and pharmacokinetics. Compared with subjects with wild-type genes, subjects with these polymorphisms require lower or higher warfarin doses to achieve adequate anticoagulant effect (12). Among these genes associated with the warfarin dose responses, cytochrome P450 2C9 (CYP2C9) and vitamin $\mathrm{K}$ epoxide reductase complex 1 (VKORC1) are two of the most prominent genes and their genetic polymorphisms could account for 30 to $40 \%$ of the warfarin dose variation $(13,14)$.

During the initiation period of warfarin therapy, patients are at greatest risk of overanticoagulation and bleeding complications, particularly in those carrying mutant CYP2C9 alleles (15). These early complications are mainly caused by inter-individual variation in response to the loading dose warfarin. Therefore, numerous warfarin-dosing algorithms 
have been developed to predict the warfarin maintenance dose and improve the effect and safety of warfarin therapy $(16,17)$. Furthermore, previous studies have mainly focused on developing a warfarin-dosing algorithm, but these algorithms also require verification and evaluation for its clinical value. In addition, polymorphism frequencies are different among ethnic groups, and compared with a Caucasian population, Chinese patients are more sensitive to warfarin (3). Therefore, it is necessary to develop a warfarin-dosing algorithm based on detected genetic polymorphisms and evaluate its clinical application in patients of the Han nationality undergoing warfarin therapy.

The present study aimed to develop a novel individualized dosing regimen based on genetic polymorphisms in CYP2C9 and VKORC1 in AF patients of Han nationality. The clinical application of this warfarin-dosing algorithm was further evaluated to predict the warfarin maintenance dose in a second unrelated population of AF patients in a randomized and controlled trial.

\section{Patients and methods}

Subjects. A total of 202 AF patients of Han nationality (111 male and 91 female, with an average age of $42.3 \pm 7.1$ years) were enrolled in the study between January 2014 and June 2015. The inclusion criteria of the study were as follows: i) Patients had received continuous warfarin therapy for $\geq 3$ month; ii) all the cases maintained a balanced diet without smoking and drinking; iii) no medications were taken that may interfere with the pharmacokinetics or pharmacodynamics of warfarin; iv) there was no hepatic or renal impairments in patients by laboratory tests; and v) no hemorrhage or thrombosis complications occurred during the warfarin therapy. Demographics of age, gender, height, body weight and maintenance doses of warfarin were collected from each AF patients. All the AF patients received chest X-ray film, electrocardiogram and echocardiography. On arrival at the hospital, plasma and blood samples were obtained. All 202 AF patients were divided into cohorts 1 (122 cases) and 2 (60 cases). Cohort 1 was used to produce the model for estimating warfarin dose, and cohort 2 was used to evaluate the feasibility of clinical application of warfarin-dosing algorithm in AF patients. The study was approved by the Ethics Committee of Shanghai Seventh People's Hospital and conducted in accordance with the Declaration of Helsinki. Written informed consent was obtained from each patient.

Determination PT and INR. Venous blood was collected from 202 enrolled AF patients in tubes containing anticoagulant $3.2 \%$ sodium citrate (the blood to anticoagulant ratio was 9:1). Centrifugation was performed at $2,000 \times \mathrm{g}$ for $10 \mathrm{~min}$ to separate plasma. The PT was measured using a Thromborel $\mathrm{S}$ reagent kit (Siemens Healthcare Diagnostic, Erlangen, Germany) on a Sysmex CA7000 analyzer (Sysmex Corp., Kobe, Japan). INR was calculated based on PT.

Genotyping of CYP2C9 and VKORC1. Peripheral venous blood was obtained from AF patients, and leukocyte genomic DNA was extracted by a DNA extraction kit (Qiagen, Crawley, UK), following the manufacturer's protocols. Subsequently, the DNA was frozen at $-20^{\circ} \mathrm{C}$ until analysis. The polymorphisms of CYP2C9 (rs1057910) and VKORC1 (rs9923231) were determined by the polymerase chain reaction-restriction fragment length polymorphism (PCR-RFLP) method, and the protocols were in accordance with the techniques described previously, with certain modifications (18). DNA samples were amplified by PCR in a final volume of $20 \mu \mathrm{l}$, containing $0.25 \mu \mathrm{M}$ primer, $0.1 \mathrm{mM}$ deoxynucleoside triphosphate (dNTP), 0.625 units Taq polymerase (Molzyme, Bremen, Germany) and $0.5 \mu \mathrm{g}$ genomic DNA in $10 \mathrm{mM}$ Tris [tris(hydroxymethyl)aminomethane]- $\mathrm{HCl}$ ( $\mathrm{pH} 9.0)$ solution (1.5 $\mathrm{mM} \mathrm{MgCl}_{2}, 50 \mathrm{mM} \mathrm{KCl}$ and $0.1 \%$ Triton $\left.\mathrm{X}-100\right)$. The PCR primers were designed and synthesized by Biomedical Engineering Co., Ltd. (Shanghai, China). Sequences for the forward and reverse primers of CYP2C9 were 5'-CACGAG GTCCAGAGATACA-3' and 5'-GGAATGAGATAGTTTCTG AATTTAAT-3', respectively. Sequences for the forward and reverse primers of VKORC1 were 5'-GCCAGCAGGAGA GGGAAATA-3' and 5'-AGTTTGGACTACAGGTGCCT-3', respectively. Cycling conditions for $\mathrm{CYP} 2 \mathrm{C} 9$ were as follows: Initial denaturation at $94^{\circ} \mathrm{C}$ for $5 \mathrm{~min}$, followed by 30 cycles of denaturation at $94^{\circ} \mathrm{C}$ for $40 \mathrm{sec}$, annealing at $56^{\circ} \mathrm{C}$ for $30 \mathrm{sec}$, and extension at $72^{\circ} \mathrm{C}$ for $30 \mathrm{sec}$, with a final extension step at $72^{\circ} \mathrm{C}$ for $10 \mathrm{~min}$. Cycling conditions for VKORC1 were as follows: Initial denaturation at $94^{\circ} \mathrm{C}$ for $5 \mathrm{~min}$, followed by 25 cycles of denaturation at $94^{\circ} \mathrm{C}$ for $1 \mathrm{~min}$, annealing at $59^{\circ} \mathrm{C}$ from $1 \mathrm{~min}$, and extension at $72^{\circ} \mathrm{C}$ for $1 \mathrm{~min}$, with a final extension step at $72^{\circ} \mathrm{C}$ for $10 \mathrm{~min}$. The amplified PCR products were incubated with restriction enzymes (Fermentas; Thermo Fischer Scientific, Waltham, MA, USA) KpnI (CYP2C9) and MspI (VKORC1) in a final volume of $30 \mu \mathrm{l}$ at $37^{\circ} \mathrm{C}$ overnight to ensure complete digestion. Finally the DNA fragments were separated by $3 \%$ agarose gel electrophoresis, and bands were stained with ethidium bromide and visualized with a UV transilluminator.

Clinical application of the warfarin-dosing algorithm. The 60 patients of cohort 2 were randomly divided into control group (30 cases) and experimental group (30 cases). In the experimental group, the genotypes of CYP2C9 and VKORC1 were determined to calculate the warfarin dose (predicted dose). Patients were administered oral warfarin based on the 'predicted dose' for 7 days, and subsequently the warfarin doses were adjusted according to the measured INR values. In the control group, warfarin was initiated at $2.5 \mathrm{mg}$ /day (standard current initiation dose of warfarin in China), and it was adjusted based on the INR values. Follow-up was carried out after initiation of warfarin therapy for 50 days, and the INR was measured daily during hospitalization. When the measured INR values of patients were within the therapeutic range of 1.8-3.0 for $\geq 7$ days, a stable warfarin maintenance dose was reached (19). The time to reach a stable warfarin maintenance dose was recorded and compared between the control and experimental groups.

Statistical analysis. All the quantitative data are expressed as mean \pm standard deviation. The commercially available software SPSS 19.0 (IBM, Corp., Armonk, NY, USA) was used to carry out statistical analysis. In the homogeneity test for variance, the unpaired Student's t-test was used to 
Table I. Characteristics of the study populations.

\begin{tabular}{|c|c|c|}
\hline Characteristics & $\begin{array}{c}\text { Cohort } 1 \\
n=122\end{array}$ & $\begin{array}{c}\text { Cohort } 2 \\
n=60\end{array}$ \\
\hline Age, years & 58.7 & 56.5 \\
\hline Male/female, $\mathrm{n}$ & $76 / 46$ & $35 / 25$ \\
\hline Height, cm & 165.6 & 165.1 \\
\hline Weight, kg & 62.1 & 63.3 \\
\hline PT, sec & 24.3 & 23.7 \\
\hline INR & 2.4 & 2.5 \\
\hline Left atrial size, mm & 45.7 & 49.7 \\
\hline Ejection fraction, $\%$ & 53.2 & 55.2 \\
\hline Daily warfarin dose, $\mathrm{mg}$ & 2.8 & 2.8 \\
\hline \multicolumn{3}{|l|}{ CYP2C9 genotype, $\mathrm{n}$} \\
\hline *1/"1 & 113 & 54 \\
\hline${ }^{*} 1 /{ }^{*} 3$ & 9 & 6 \\
\hline \multicolumn{3}{|l|}{ VKORC1 genotype, n } \\
\hline AA & 105 & 49 \\
\hline $\mathrm{AG}$ & 15 & 10 \\
\hline GG & 2 & 1 \\
\hline
\end{tabular}

PT, prothrombin time; INR, international normalized ratio; CYP2C9, cytochrome P450 2C9; VKORC1, vitamin K epoxide reductase complex 1 .

compare the quantitative data and Fisher's exact test was used to compare the enumeration data. The Kaplan-Meier method was performed to analyze the time interval between initiation of warfarin therapy and warfarin maintenance dose, and their difference between the control and experimental groups was compared with the log-rank test. Pearson's correlation test was performed to analyze the linear correlation between predicted dose and actual dose of warfarin. The associations between warfarin dose and other variables were analyzed by multivariate linear regression and to develop a novel warfarin-dosing algorithm. $\mathrm{P}<0.05$ was considered to indicate a statistically significant difference.

\section{Results}

Patient characteristics. There was a total of 202 AF patients of Han nationality who participated in the study. Among them, 122 patients with stable control of anticoagulation were recruited to develop warfarin-dosing algorithm based on the CYP2C9 and VKORC1 genotypes (cohort 1), and 60 patients were recruited to evaluate the clinical application of warfarin-dosing algorithm (cohort 2). The patient demographics of age, gender, height, weight, PT, INR, left atrial size, ejection fraction, warfarin dose requirements and genotype are shown in Table I.

CYP2C9 and VKORC1 genotyping. All 122 samples were genotyped for the CYP2C9 (rs1057910) and VKORC1 (rs9923231), and these two polymorphisms previously have been reported to affect warfarin-dose requirement. There were 113 subjects

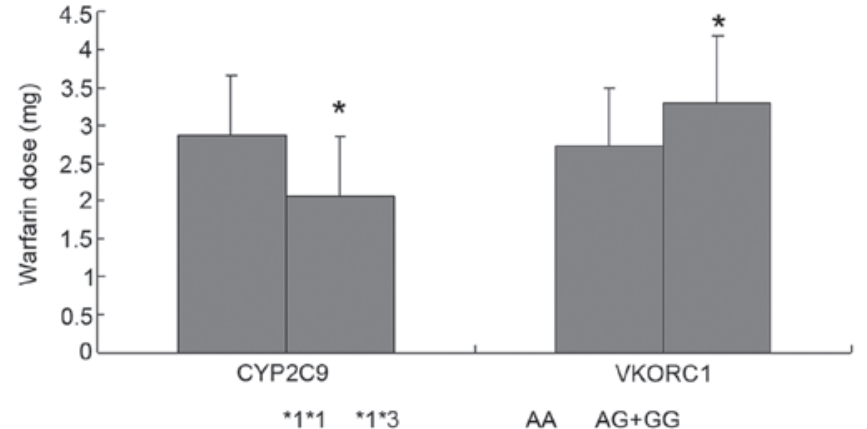

Figure 1. Warfarin dose in different cytochrome P450 2C9 (CYP2C9) and vitamin $\mathrm{K}$ epoxide reductase complex 1 (VKORC1) genotypes. All data are expressed as the mean values \pm standard deviation. Significant difference from the ${ }^{*} 1 /{ }^{*} 1$ or AA genotype, ${ }^{*} \mathrm{P}<0.05$.

with homozygous CYP2C9*1/*1 and 9 subjects with heterozygous CYP2C9*1/33. For VKORC1 genotyping, there were 105 subjects with homozygous AA genotype, 15 subjects with heterozygous AG genotype and 2 subjects with homozygous GG genotype. The observed genotype frequency of CYP2C9 and VKORC1 showed no deviation from Hardy-Weinberg equilibrium $(\mathrm{P}>0.05)$.

Associations of warfarin dose with CYP2C9 and VKORC1. The mean warfarin daily dose requirement was $2.06 \pm 0.79 \mathrm{mg}$ in heterozygous CYP2C9*1/3 patients, which was significantly lower than that in homozygous wild-type CYP2C9*1/*1 patients $(2.88 \pm 0.77 \mathrm{mg}, \mathrm{P}<0.05)$. The mean warfarin daily dose requirement was $3.30 \pm 0.87 \mathrm{mg}$ in patients with the VKORC1 AG and GG genotype, which was significantly higher than that in patients with AG genotype $(2.74 \pm 0.76 \mathrm{mg}, \mathrm{P}<0.05)$ (Fig. 1). The multivariate regression analysis was performed to detect variables influencing warfarin dose. The results showed that age, height, and CYP2C9 and VKORC1 genotypes can develop models for estimating warfarin dose, and the best warfarin-dosing algorithm has the largest $\mathrm{R}^{2}$ value (Table II).

Clinical application of warfarin-dosing algorithm in AF patients. The warfarin-dosing algorithm was further evaluated in a second unrelated population of AF patients, who were treated with oral warfarin and with stable control of anticoagulation (cohort 2). The patient characteristics and demographics of cohort 2 are shown in Table I. There were no significant differences in age, gender, height, weight, PT, INR, left atrial size, ejection fraction and warfarin dose requirements between cohorts 1 and 2. No significant differences were identified in the frequency of the CYP2C9 or VKORC1 genotypes between the two cohorts. The 60 patients of cohort 2 were randomly divided into control group (30 cases received $2.5 \mathrm{mg} /$ day of oral warfarin for the first 7 days) and experimental group (30 cases received oral warfarin based on the 'predicted dose' from warfarin-dosing algorithm for the first 7 days), and subsequently warfarin doses were adjusted according to INR values. The two groups showed homogeneity in age, gender, height, weight, PT, INR, left atrial size and ejection fraction (Table III). During the 50-day follow-up, 19 patients $(63.3 \%)$ in the control group and 26 patients $(86.7 \%)$ in the experimental group received the warfarin maintenance dose, and patients with the 
Table II. Regression equation for modeling warfarin daily dose requirements based on age, height and genotype.

\begin{tabular}{llll}
\hline Model, X variables & \multicolumn{1}{c}{ Regression equation } & P-value & $\mathrm{R}^{2}$ for model, $\%$ \\
\hline Age, years & $\mathrm{y}=2.81-0.0132$ (age) & $<0.01$ & 15.8 \\
Height & $\mathrm{y}=2.17+0.0227$ (height) & $<0.01$ & 14.2 \\
CYP2C9 genotype & $\mathrm{y}=2.01-0.371(\mathrm{CYP} 3)$ & $<0.01$ & 18.7 \\
VKORC1 genotype & $\mathrm{y}=1.43+0.274($ VKORC1) & $<0.01$ & 17.1 \\
Age, height, and CYP2C9 & Dose=0.438-0.0143 (age)+0.0149 (height)- & $<0.01$ & 56.4 \\
and VKORC1 genotypes & $0.324\left(\mathrm{CYP}^{*} 3\right)+0.209$ (VKOR) & &
\end{tabular}

Age: Input age in years; height: Input height in centimeters; CYP2C9 genotype: Input 0 or 1 for the number of * 3 alleles within the patient's genotype; VKORC1 genotype: Input 0 for AA, 1 for AG, and 2 for GG. CYP2C9, cytochrome P450 2C9; VKORC1, vitamin K epoxide reductase complex 1 .

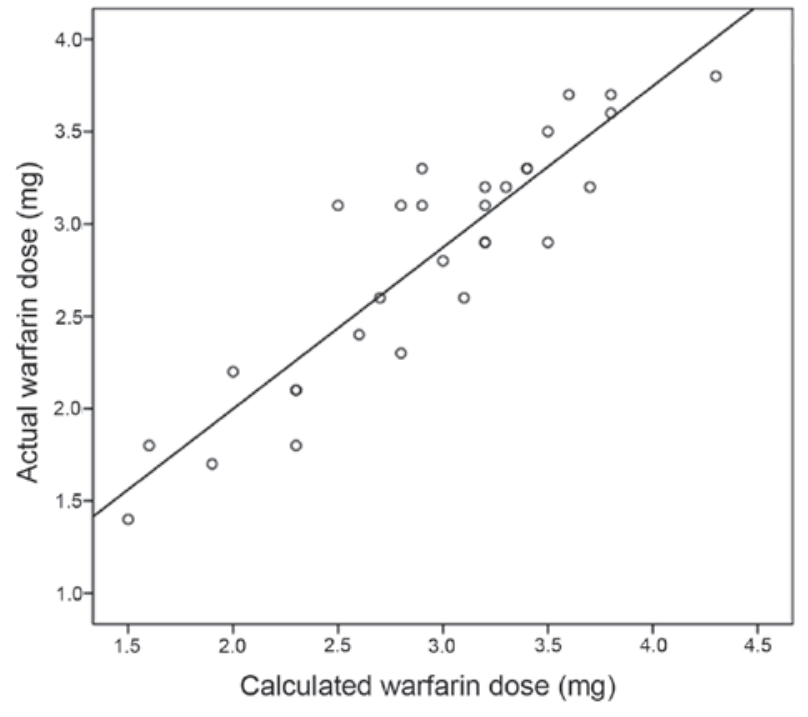

Figure 2. Correlation analysis of calculated warfarin dose using a warfarin-dosing algorithm and actual dose in unrelated cohort 2 of atrial fibrillation patients. Each patient within the second cohort is represented by a circle.

predicted warfarin dose showed more cases acquiring warfarin maintenance dose $(\mathrm{P}<0.05)$. Among all 45 patients who reached the stable warfarin maintenance dose, patients in the experimental group had a shorter time elapse from initiation until stable warfarin maintenance dose $(25.8 \pm 1.7$ days $)$ than that in the control group $(33.1 \pm 1.9$ days $)(\mathrm{P}<0.05)$ (Table III). In these 45 patients, Pearson's correlation analysis showed a significant correlation between the calculated warfarin dose using the warfarin-dosing algorithm and actual dose ( $\mathrm{r}=0.822$; $\mathrm{P}<0.01$ ) (Fig. 2).

\section{Discussion}

AF is the one common cardiac arrhythmia in the clinical setting with substantial morbidity and mortality. AF patients have an increased risk of stroke and systemic thromboembolism (20). The risk of complications in AF can be reduced by anticoagulant therapy, such as warfarin (21). However, during the initiation period of warfarin therapy, patients have a higher incidence of overanticoagulation and resultant bleeding. Due to the inter-individual variability in response to warfarin, it
Table III. Characteristics of the study populations in cohort 2 .

\begin{tabular}{lcc}
\hline Characteristics & $\begin{array}{c}\text { Control group, } \\
\mathrm{n}=30\end{array}$ & $\begin{array}{c}\text { Experimental } \\
\text { group, } \mathrm{n}=30\end{array}$ \\
\hline Age, years & 57.0 & 56.1 \\
Male/female, $\mathrm{n}$ & $76 / 46$ & $35 / 25$ \\
Height, cm & 165.0 & 165.3 \\
Weight, kg & 62.1 & 63.3 \\
PT, sec & 23.4 & 24.0 \\
INR & 2.6 & 2.4 \\
Left atrial size, mm & 49.2 & 50.2 \\
Ejection fraction, \% & 54.5 & 55.8 \\
CYP2C9 genotype, $\mathrm{n}$ & & 27 \\
${ }^{*} 1{ }^{*} 1$ & 27 & 3 \\
${ }^{*} 1$ \% $^{*} 3$ & 3 & \\
VKORC1 genotype, $\mathrm{n}$ & & 25 \\
AA & 26 & 5 \\
AG & 5 & $25.8 \pm 1.7^{\mathrm{a}}$ \\
GG & 1 & $(\mathrm{n}=26)$ \\
Time for obtaining stable & $33.1 \pm 1.9$ & \\
warfarin maintenance & $(\mathrm{n}=19)$ & \\
dose (day) & & \\
\hline
\end{tabular}

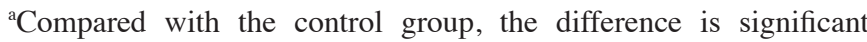
$(\mathrm{P}<0.05)$.

is difficult to makes accurate dose predication. A number of factors, such as patient demographics, environment, clinical background and genetic polymorphism, contribute to the inter-individual variation of the warfarin dose response $(9,10)$. Among them, genetic polymorphisms in genes are associated with the warfarin pharmacodynamics, and pharmacokinetics have increasingly important roles in predicting warfarin dose.

In the present study, the genotypes of CYP2C9 and VKORC1 were determined in 122 AF patients undergoing warfarin therapy. The results showed that warfarin dose could be estimated by variables, such as age, height, and the CYP2C9 and VKORC1 genotypes. A new warfarin-dosing 
algorithm was also developed and its validity was confirmed in a second cohort of AF patients. Compared with the control group, patients with a predicted dose from the warfarin-dosing algorithm showed more patients acquiring the warfarin maintenance dose during the 50-day follow-up, a significantly shorter time elapse from initiation until warfarin maintenance dose. Among all the patients acquiring the warfarin maintenance dose, the predicted warfarin dose was significantly correlated with the actual dose.

CYP2C9 is one isoform of the cytochrome P450 complex and participates in the metabolism of $\sim 15 \%$ of the clinically used drugs, including non-steroidal anti-inflammatory drugs, angiotensin II blockers and oral anticoagulants (22). CYP2C9 is located on chromosome 10q24.2, and its alleles have been identified. The wild-type allele CYP2C9*1 shows normal enzyme activity and a high allelic frequency. The two allelic variants CYP2C9*2 and *3 have allelic frequencies of $\sim 13$ and $7 \%$, and lower enzyme activity in comparison to the wild-type (CYP2C9*1) (23). In the present study, the allelic variant CYP2C9*2 was not detected and is uncommon in the Han population. CYP2C9*3 (rs1057910) is located at amino acid position 359 in exon 7 , and is defined by an A-to-C nucleotide substitution (1075 A>C), resulting in a non-synonymous substitution amino acid change from leucine to isoleucine. The allelic frequency of CYP2C9*3 in this study was $7.8 \%$, and is similar with previous studies, who showed $4.5 \%$ allelic frequency of CYP2C9*3 in the Chinese population (24). The present study also identified that subjects with the CYP2C9*1/*3 genotype had a lower mean warfarin daily dose requirement compared with subjects with wild-type CYP2C9*1/*1. This suggests that in CYP2C9*1/*3 genotype subjects, the enzyme activity was reduced and metabolism and clearance of warfarin was delayed, thus enhancing their sensitivity to warfarin and decreasing the warfarin daily dose requirement.

VKORC1 is responsible for the transformation of epoxide (vitamin K-2,3-epoxide) to its reduced form (vitamin K hydroquinone), this process is required for the $\gamma$-carboxylation of the vitamin K-dependent coagulation factors (factors II, VII, IX and X). Warfarin inhibits the above process catalyzed by the VKORC1 enzyme complex, thus preventing the regeneration of the reduced form of vitamin K (25). Mutations in VKORC1 can lead to warfarin resistance $(26,27)$. rs9923231 $(-1639 \mathrm{G}>\mathrm{A})$ is the most common SNP of VKORC1 gene. In the Caucasian population, GG is the highest genotype and AA is the lowest (3). However, in the Chinese population, AA is the highest genotype and its frequency is $>83 \%(24,28)$. The present study confirmed this finding and detected 115 subjects with the AA genotype in 122 cases. Furthermore, subjects with the AG+GG genotype of VKORC1 had a higher mean warfarin daily dose requirement than subjects with wild-type AA genotype. This indicates that the $\mathrm{G}$ allele produces VKORC1 with a lower activity and results in warfarin resistance and a higher warfarin daily dose requirement. The present results are in accordance with another study showing that subjects with the GG genotype showed a higher warfarin dose compared to the subjects with the AG or AA genotype (29).

The present study showed that the warfarin maintenance dose can be best estimated by variables, such as age, height, and CYP2C9 and VKORC1 genotypes. A dosing algorithm was developed and its efficacy was further validated in an unrelated cohort of AF patients undergoing warfarin therapy. A prospective clinical study was performed to assess the dosing algorithm in the initiation stage of warfarin therapy in AF patients. During the 50-day follow-up, more patients received the warfarin maintenance dose in the experimental group (86.7\%) compared to the control group (63.3\%). Among all the patients acquiring the warfarin maintenance dose, Pearson's correlation analysis showed that the predicted dose was prominently correlated with the actual dose, making effective prediction of the actual warfarin maintenance dose by the warfarin-dosing algorithm. The predicted warfarin maintenance dose, which is based on a combination of genetic factors and non-genetic factors of AF patients, could effectively reduce the time elapse from initiation until stable warfarin maintenance dose. Due to the limited cases and observation time, our warfarin-dosing algorithm requires further investigation and clinical application. With more warfarin-related genes identified and confirmed, and more non-genetic factors revealed, the warfarin maintenance dose predicted from the algorithm would be more accurate, thereby improving the efficacy and safety of warfarin therapy.

In conclusion, a new warfarin-dosing algorithm was developed based on age, height, and CYP2C9 and VKORC1 genotypes, and it can shorten the time elapse from initiation until warfarin maintenance dose in AF patients. This warfarin-dosing algorithm can promote patients acquiring warfarin maintenance dose during the follow-up, and shorten the time elapse from initiation until warfarin maintenance dose. Further study is required to expand the sample size and assess the clinical application in AF patients undergoing warfarin therapy.

\section{Acknowledgements}

The present study was funded by the Pudong Health and Family Planning Commission (grant no. PW2013A-24) and the Talents Training Program of Shanghai Seventh People's Hospital (grant no. XX2013-001)

\section{References}

1. Ansell J, Hirsh J, Poller L, Bussey H, Jacobson A and Hylek E: The pharmacology and management of the vitamin $\mathrm{K}$ antagonists: The Seventh ACCP Conference on Antithrombotic and Thrombolytic Therapy. Chest 126 (Suppl 3): 204S-233S, 2004.

2. Hirsh J, Guyatt G, Albers GW and Schunemann HJ: Proceedings of the Seventh ACCP Conference on Antithrombotic and Thrombolytic Therapy: Evidence-based guidelines. Chest 126 (Suppl 3): 172S-696S, 2004.

3. Lam MP and Cheung BM: The pharmacogenetics of the response to warfarin in Chinese. Br J Clin Pharmacol 73: 340-347, 2012.

4. Hawes EM and Viera AJ: Anticoagulation: Monitoring of patients receiving anticoagulation. FP Essent 422: 24-30, 2014.

5. No authors listed: Effect of long-term oral anticoagulant treatment on mortality and cardiovascular morbidity after myocardial infarction. Anticoagulants in the Secondary Prevention of Events in Coronary Thrombosis (ASPECT) Research Group. Lancet 343: 499-503, 1994.

6. Cannegieter SC, Rosendaal FR, Wintzen AR, van der Meer FJ, Vandenbroucke JP and Briët E: Optimal oral anticoagulant therapy in patients with mechanical heart valves. N Engl J Med 333: 11-17, 1995.

7. Fihn SD, McDonell M, Martin D, Henikoff J, Vermes D, Kent D and White RH; Warfarin Optimized Outpatient Follow-up Study Group: Risk factors for complications of chronic anticoagulation. A multicenter study. Ann Intern Med 118: 511-520, 1993. 
8. No authors listed: Adjusted-dose warfarin versus low-intensity fixed-dose warfarin plus aspirin for high-risk patients with atrial fibrillation: Stroke prevention in atrial fibrillation III randomised clinical trial. Lancet 348: 633-638, 1996.

9. Hirsh J, Dalen J, Anderson DR, Poller L, Bussey H, Ansell J and Deykin D: Oral anticoagulants: Mechanism of action, clinical effectiveness, and optimal therapeutic range. Chest 119 (Suppl 1): 8S-21S, 2001.

10. Takahashi $\mathrm{H}$ and Echizen $\mathrm{H}$ : Pharmacogenetics of warfarin elimination and its clinical implications. Clin Pharmacokinet 40 587-603, 2001.

11. Approves Updated Warfarin FDA: (Coumadin) Prescribing information: New genetic information may help providers improve initial dosing estimates of the anticoagulant for individual patients. Available from http://www.fda.gov/bbs/topics/NEWS/2007/NEW01684.html. Accessed February 19, 2009.

12. MazzaccaraC,ContiV,LiguoriR,SimeonV,TorielloM,SeveriniA, Perricone C, Meccariello A, Meccariello P, Vitale DF, et al: Warfarin anticoagulant therapy: A Southern Italy pharmacogenetics-based dosing model. PLoS One 8: e71505, 2013.

13. Bodin L, Verstuyft C, Tregouet DA, Robert A, Dubert L, Funck-Brentano C, Jaillon P, Beaune P, Laurent-Puig P, Becquemont L, et al: Cytochrome P450 2C9 (CYP2C9) and vitamin $\mathrm{K}$ epoxide reductase (VKORC1) genotypes as determinants of acenocoumarol sensitivity. Blood 106: 135-140, 2005

14. Vecsler M, Loebstein R, Almog S, Kurnik D, Goldman B, Halkin $\mathrm{H}$ and Gak E: Combined genetic profiles of components and regulators of the vitamin K-dependent gamma-carboxylation system affect individual sensitivity to warfarin. Thromb Haemost 95: 205-211, 2006.

15. Aithal GP, Day CP, Kesteven PJL and Daly AK: Association of polymorphisms in the cytochrome P450 CYP2C9 with warfarin dose requirement and risk of bleeding complications. Lancet 353 : 717-719, 1999.

16. Ekladious SM,Issac MS,El-Atty Sharaf SA and Abou-YoussefHS: Validation of a proposed warfarin dosing algorithm based on the genetic make-up of Egyptian patients. Mol Diagn Ther 17: 381-390, 2013

17. Li X, Liu R, Luo ZY, Yan H, Huang WH, Yin JY, Mao XY, Chen XP, Liu ZQ, Zhou HH, et al: Comparison of the predictive abilities of pharmacogenetics-based warfarin dosing algorithms using seven mathematical models in Chinese patients. Pharmacogenomics 16: 583-590, 2015.
18. Scibona P, Redal MA, Garfi LG, Arbelbide J, Argibay PF and Belloso WH: Prevalence of CYP2C9 and VKORC1 alleles in the Argentine population and implications for prescribing dosages of anticoagulants. Genet Mol Res 11: 70-76, 2012.

19. Voora D, Eby C, Linder MW, Milligan PE, Bukaveckas BL, McLeod HL, Maloney W, Clohisy J, Burnett RS, Grosso L, et al: Prospective dosing of warfarin based on cytochrome P-450 2C9 genotype. Thromb Haemost 93: 700-705, 2005.

20. Senoo K, Lane D and Lip GY: Stroke and bleeding risk in atrial fibrillation. Korean Circ J 44: 281-290, 2014.

21. Lip GY and Lane DA: Stroke prevention in atrial fibrillation: A systematic review. JAMA 313: 1950-1962, 2015.

22. Zhou SF, Zhou ZW and Huang M: Polymorphisms of human cytochrome P450 2C9 and the functional relevance. Toxicology 278 : 165-188, 2010.

23. Vogl S, Lutz RW, Schönfelder G and Lutz WK: CYP2C9 genotype vs. metabolic phenotype for individual drug dosing - a correlation analysis using flurbiprofen as probe drug. PLoS One 10: e0120403, 2015.

24. Li S, Zou Y, Wang X, Huang X, Sun Y, Wang Y, Dong L and Jiang H: Warfarin dosage response related pharmacogenetics in Chinese population. PLoS One 10: e0116463, 2015.

25. Oldenburg J, Watzka M, Rost S and Müller CR: VKORC1: Molecular target of coumarins. J Thromb Haemost 5 (Suppl 1); $1-6,2007$

26. Rost S, Fregin A, Ivaskevicius V, Conzelmann E, Hörtnagel K, Pelz HJ,Lappegard K, Seifried E, Scharrer I, TuddenhamEG, et al: Mutations in VKORC1 cause warfarin resistance and multiple coagulation factor deficiency type 2. Nature 427: 537-541, 2004.

27. Harrington DJ, Gorska R, Wheeler R, Davidson S, Murden S, Morse C, Shearer MJ and Mumford AD: Pharmacodynamic resistance to warfarin is associated with nucleotide substitutions in VKORC1. J Thromb Haemost 6: 1663-1670, 2008.

28. Yang L, Ge W, Yu F and Zhu H: Impact of VKORC1 gene polymorphism on interindividual and interethnic warfarin dosage requirement--a systematic review and meta analysis. Thromb Res 125: e159-e166, 2010.

29. Sconce EA, Khan TI, Wynne HA, Avery P, Monkhouse L, King BP, Wood P, Kesteven P, Daly AK and Kamali F: The impact of CYP2C9 and VKORC1 genetic polymorphism and patient characteristics upon warfarin dose requirements: Proposal for a new dosing regimen. Blood 106: 2329-2333, 2005. 